\title{
A numerical analysis of seismic waves for an anisotropic fault zone
}

\author{
Takeshi Nakamura* and Hiroshi Takenaka \\ Department of Earth and Planetary Sciences, Kyushu University, Hakozaki 6-10-1, Fukuoka 812-8581, Japan
}

(Received January 24, 2005; Revised January 4, 2006; Accepted January 31, 2006; Online published May 12, 2006)

\begin{abstract}
In this study we examine the effects of anisotropy on the seismic wavefield in a fault zone from computation of the synthetic seismograms for a simple fault zone model and a variety of seismic wave sources. The fault zone is modeled by a homogeneous vertical layer with transverse isotropy, induced by cracks, sandwiched between isotropic half-spaces (host rocks). The symmetry axis of the transverse isotropy is horizontal and perpendicular to the fault zone strike. We calculate the synthetic seismograms for this anisotropic fault zone model using a semianalytical method, the propagator matrix method. The synthetic seismograms show a later phase arriving after the main shear-wave in the horizontal component perpendicular to the fault zone strike at most stations near the fault zone. It is the slower shear-wave $\left(q S_{2}\right)$ and its reverberation. The amplitude of this phase and the time delay from the main shear-wave arrival are proportional to the degree of anisotropy, which suggests that observing such phase in field measurements may imply the presence of an anisotropic fault zone. We also perform the shear-wave splitting measurements by applying the cross-correlation method to the synthetic seismograms for various sources. For a strike-slip source, the synthetic seismograms show that the wavefield is more affected by the velocity structure than by the degree of anisotropy, which makes it difficult to estimate the anisotropic (shearwave splitting) parameters. For normal and dip-slip fault sources with the strike parallel to or striking against the fault zone, the effects of anisotropy is so dominant that the anisotropic fault zone can be detected. These results suggest that the determination of the anisotropic properties in the fault zone would require an appropriate station deployment and the source type information.
\end{abstract}

Key words: Seismic anisotropy, shear-wave splitting, synthetic seismogram, fault zone.

\section{Introduction}

Seismic anisotropy is one of the many physical properties describing the Earth's internal structure. The presence of anisotropy causes the changing of arrival time, propagation direction, amplitude and phase of the seismic wavefield. Since 1980, studies on seismic anisotropy have revealed spatial and temporal variations in stress fields, cracks, fracturing, crystals, fluid flowing, and so on (e.g., Crampin et al., 1980; Anderson and Dziewonski, 1982; Peacock et al., 1988; Savage, 1999). These previous studies have significantly contributed to our understanding the Earth dynamics, and anisotropy is currently an interesting part in seismology.

Anisotropy produces two major effects on the seismogram. One is the azimuthal travel time variation with the propagation direction of seismic waves, and the other is the shear-wave splitting (Crampin, 1978) where a shear-wave is split into two orthogonal polarizations, called $q S_{1}$ - and $q S_{2}$-waves, which travel at different speeds. The first effect is difficult to recognize in the seismogram because of interference (noise or other phases), but the second effect

\footnotetext{
* Now at Marine Technology Center, Japan Agency for Marine-Earth Science and Technology, Natsushima-cho 2-15, Yokosuka 237-0061, Japan.

Copyright (c) The Society of Geomagnetism and Earth, Planetary and Space Sciences (SGEPSS); The Seismological Society of Japan; The Volcanological Society of Japan; The Geodetic Society of Japan; The Japanese Society for Planetary Sciences; TERRAPUB.
}

can be recognized easily even at a single station. Thus, a number of studies have reported seismic anisotropy from measuring the shear-wave splitting. Recent studies based on these measurements using a dense seismic array have allowed us to image anisotropic structures around fault zones (e.g., Cochran et al., 2003).

The fault zone is thought to be a highly damaged area (Chester et al., 1993), and the seismic wave propagation in the fault zone, where the trapped wave and the fault zone head wave are generated, appears to be significantly different from that in the surrounding rock (e.g., Li et al., 1990; Hough et al., 1994). This intensive damaging causes velocity reduction and also anisotropy, and in some cases the degree of anisotropy in the fault zone is higher than that of the surrounding rocks (e.g., Mizuno et al., 2001; Watanabe et al., 2001). Nakamura et al. (2005) have found the spatial change of anisotropy in and around the aftershock region from analyzing the shear-wave splitting recorded near the source fault of the 2000 Tottori-ken Seibu earthquake Mw6.6. Peng and Ben-Zion (2004) have performed automatic measurements of the shear-wave splitting for about 22000 recordings in the North Anatolian fault and suggested that the anisotropy is confined to the top 3-4 km of the crust.

Most of the shear-wave splitting measurements assuming a homogeneous layer have not been taken into account the effects of the velocity structure around the fault zone. Since the shear-waves are distorted by the interaction with the interfaces between the fault zone and the surround- 


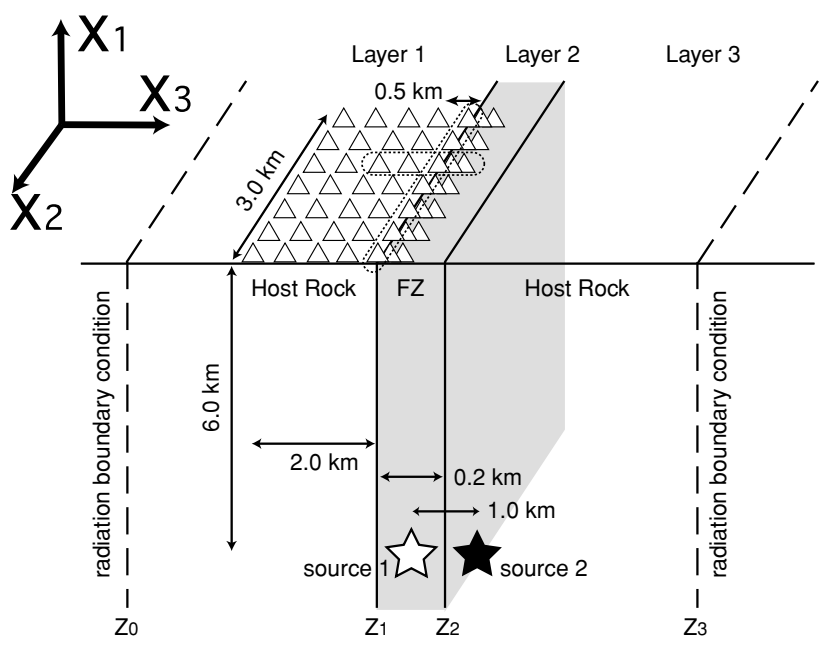

Source Type

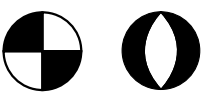

strike slip normal fault

(SS-A) (NF-A)
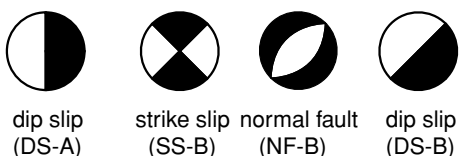

Fig. 1. Overview of simulation model: sources (stars), stations (triangles), and the fault zone (shaded). Stations inside and outside the fault zone are configured at an interval of $0.04 \mathrm{~km}$ and $0.5 \mathrm{~km}$ along the axis $x_{3}$ from the interface, respectively. Source mechanisms used in this study are also shown. We show seismograms at stations marked by dotted lines in Fig. 2.

Table 1. Isotropic properties of the structure model. The velocities of $P$ and $S$-waves and the density are expressed by $\alpha, \beta$ and $\rho$, respectively.

\begin{tabular}{c|c|c|c|c}
\hline Layer & $\begin{array}{c}\alpha \\
(\mathrm{km} / \mathrm{s})\end{array}$ & $\begin{array}{c}\beta \\
(\mathrm{km} / \mathrm{s})\end{array}$ & $\begin{array}{c}\rho \\
\left(\mathrm{g} / \mathrm{cm}^{3}\right)\end{array}$ & $\begin{array}{c}\text { thickness } \\
(\mathrm{km})\end{array}$ \\
\hline \hline 1 & 5.55 & 3.20 & 2.50 & \\
2 & 4.85 & 2.80 & 2.50 & 0.2 \\
3 & 5.55 & 3.20 & 2.50 & \\
\hline
\end{tabular}

ing rock, measuring the shear-wave splitting could sometimes raise problems with incorrect measurement results and lead to misinterpretation of subsurface features if particular attentions on structure are not paid. Liu and Crampin (1990) have examined the effects on shear-wave propagating through both cases of horizontal isotropic-to-isotropic and isotropic-to-anisotropic interfaces. Nakamura and Takenaka (2005) have examined the accuracy of measuring shear-wave splitting around the fault zone with anisotropy due to cracks aligned in a preferred direction parallel to the strike of a fault zone. They suggested that measuring shear-wave splitting is difficult for a pure strike slip source because the influence of the velocity contrast is dominant. They did not investigate any cases for other types of source. In this paper, we examine various types of sources and many stations deployed around the fault zone by computing the synthetic seismograms for the anisotropic fault zone model.

\section{Fault Zone Model}

We consider a homogeneous vertical layer (fault zone) with transverse isotropy (hexagonal anisotropy), induced by cracks or fractures, sandwiched between isotropic halfspaces. The transverse isotropy exhibits symmetry about one of horizontal axes $x_{3}$ (Fig. 1), and the speeds of propagation depend on the angle between the propagation direction and the symmetry axis. We take the vertical axis $x_{1}$ positive upward and the $x_{2}-x_{3}$ plane horizontal plane. We use a one-dimensional structure along the $x_{3}$ direction in which the density and velocities are vertically layered, as shown in Fig. 1. The model of the fault zone is of $200 \mathrm{~m}$ width, similar to the model used by Igel et al. (1997). Table 1 shows the material parameters of the isotropic model.

We calculate the elastic constants in anisotropy in the fault zone from Hudson's $(1980,1981)$ crack model. In this model, the elastic constants are given in terms of the propagation shear-wave velocity in isotropy (Table 1), the aspect ratio of the crack and the crack density $\varepsilon$ defined by O'Connell and Budiansky (1974). The crack density $\varepsilon$ is a non-dimensional value given by the number of cracks with size per unit volume and is approximately equal to a hundredth of the percentage of differential shear-wave velocity anisotropy. Observed shear-wave splitting suggests that most rocks are close to the critical state on fracturing around $\varepsilon=0.05$ where cracks are so closely spaced that shear-strength is lost and earthquakes can occur (e.g., Crampin and Peacock, 2005). Thus, referring to this value, in this paper we consider three cases of anisotropy with $\varepsilon=0.02,0.06$ and 0.1 , assuming fluid-filled thin cracks with aspect ratio 0.0001 which are aligned to the strike of the fault zone. Table 2 shows the values of anisotropic velocities in the fault zone.

The source types considered here are strike-slip (hereafter called SS), normal (NF) and dip-slip (DS) fault, represented by double-couple point sources (Fig. 1). The fault strike for each source type is chosen to be parallel to that of the fault zone or $45^{\circ}$ striking (hereafter called $* *_{-}$A and $* *_{\text {- }}$ $\mathrm{B}$ type, respectively). The source position is at the center of the fault zone or $1 \mathrm{~km}$ offset from the center. The recording stations are deployed inside and outside the fault zone in a horizontal plane with the vertical offset of $6 \mathrm{~km}$ from the source (see Fig. 1).

\section{Numerical Method}

We exploit Nakamura and Takenaka's (2005) approach, which adapts Mandal and Mitchell's (1986) method based on the propagator-matrix (Haskell, 1953; Gilbert and Backus, 1966) to calculate synthetic seismograms for transversely isotropic layered media. Mandal and Mitchell's (1986) method takes the symmetry axis $\left(x_{3}\right)$ along the vertical direction and calculate the displacements at the free surface, while Nakamura and Takenaka's (2005) approach takes the symmetry axis in the horizontal direction perpendicular to the fault zone (Fig. 1). Associated with this coordinate transform, the boundary condition at the first boundary (plane $x_{3}=z_{0}$ in Fig. 1) is changed from the free surface condition into the radiation condition (see Figs. A1 and A2). This implementation in the propagator matrix technique is described in Appendix A. In this approach, although the exact free surface condition cannot be set at any horizontal planes (i.e. $x_{1}-x_{2}$ plane), the effects of free surface can be approximately incorporated in the syn- 

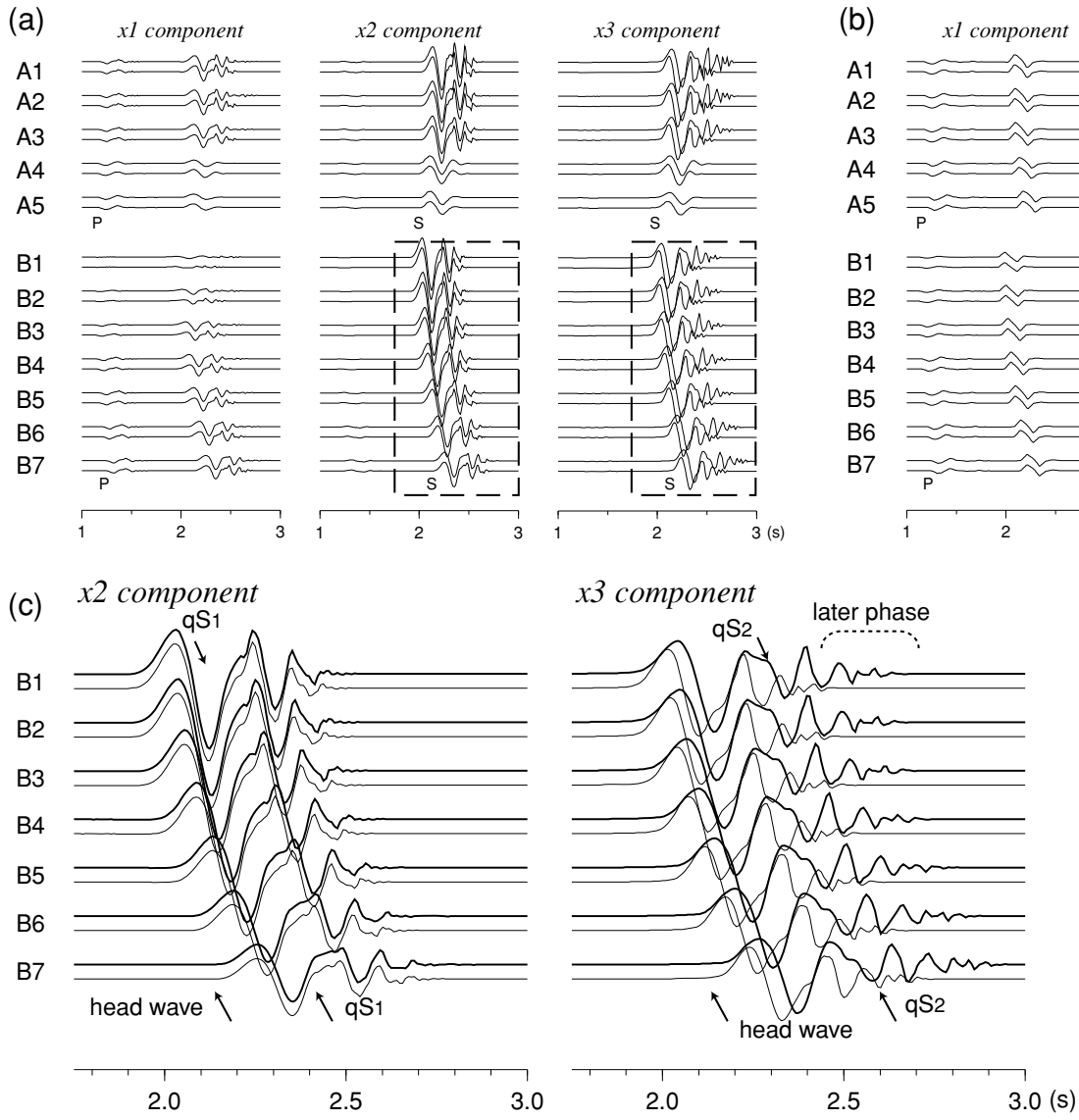

(b)

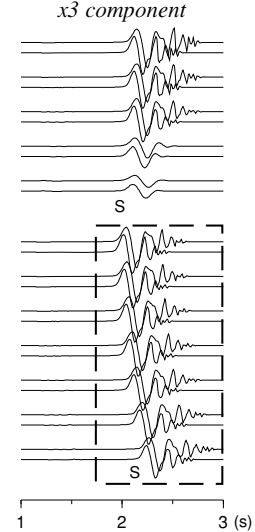

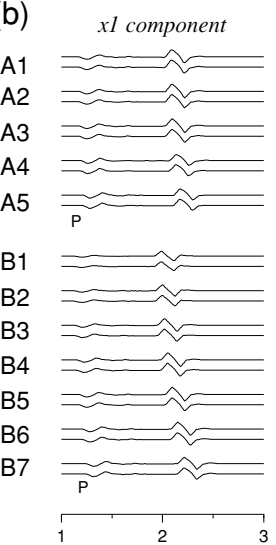
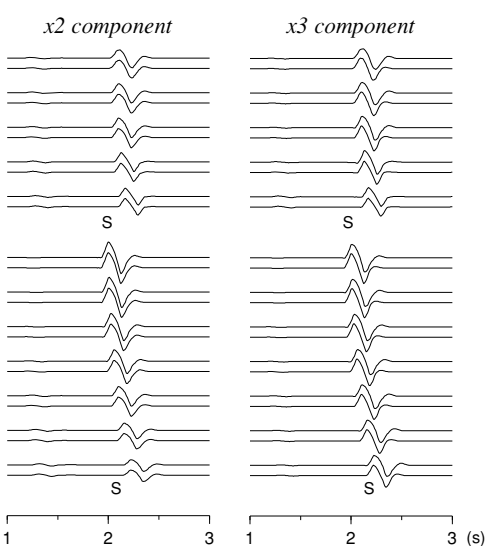

(d)

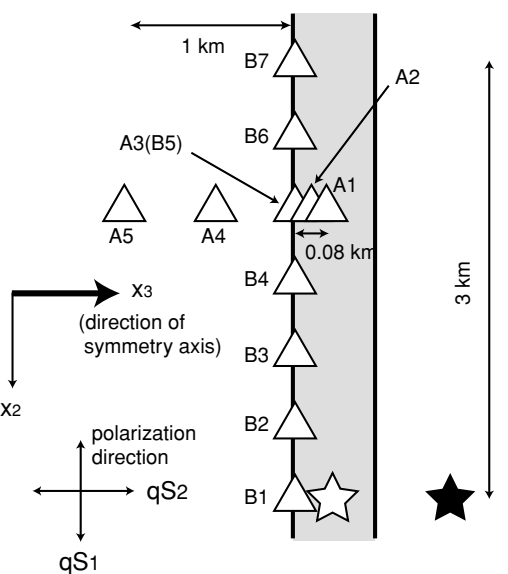

Fig. 2. Synthetic seismograms. Upper and lower traces at each stations show the seismogram through the anisotropic $(\varepsilon=0.06)$ and isotropic $(\varepsilon=0.0)$ fault zone, respectively. (a) Synthetic seismograms for DS-B source located inside the fault zone. Note that the amplitudes of seismogram are half compared with those at stations at the free surface. (b) Synthetic seismograms for DS-B source located outside the fault zone. (c) Enlarged seismograms in $x_{2}$ and $x_{3}$ component at stations B1-B7 as shown by a dashed rectangle in Fig. 2(a). The onsets of the head wave and the direct $q S_{1}$ and $q S_{2}$ phases are indicated by arrows. (d) Station name for each seismogram and location to the fault zone used in this study. The direction of symmetry axis of anisotropy and the polarization direction of $q S_{1}$ and $q S_{2}$ are also shown.

Table 2. Anisotropic properties in the fault zone and the source location for each case of simulation. The aspect ratios used in this study is 0.0001 . The velocities of horizontal and vertical $P$ - and $S$-waves are expressed by elastic constants and density: $\alpha_{H}=\sqrt{C_{11} / \rho}$, $\alpha_{V}=\sqrt{C_{33} / \rho}, \beta_{V}=\sqrt{C_{44} / \rho}, \beta_{H}=\sqrt{C_{66} / \rho}$. Another velocity parameter $\eta$ is represented by $\eta=\sqrt{C_{12} / \rho}$. In Hudson's $(1980,1981)$ model, $C_{66}$ is independent of the crack density.

\begin{tabular}{c|c|c|c|c|c|c|c}
\hline Case & $\begin{array}{c}\text { Source } \\
\text { position }\end{array}$ & $\varepsilon$ & $\begin{array}{c}\alpha_{H} \\
(\mathrm{~km} / \mathrm{s})\end{array}$ & $\begin{array}{c}\alpha_{V} \\
(\mathrm{~km} / \mathrm{s})\end{array}$ & $\begin{array}{c}\beta_{V} \\
(\mathrm{~km} / \mathrm{s})\end{array}$ & $\begin{array}{c}\beta_{H} \\
(\mathrm{~km} / \mathrm{s})\end{array}$ & $\begin{array}{c}\eta \\
(\mathrm{km} / \mathrm{s})\end{array}$ \\
\hline \hline 1 & & 0.02 & 4.85 & 4.85 & 2.74 & & 2.80 \\
2 & $\mathrm{FZ}$ & 0.06 & 4.85 & 4.85 & 2.61 & 2.80 & 2.80 \\
3 & & 0.1 & 4.85 & 4.85 & 2.50 & & 2.80 \\
\hline 4 & & 0.02 & 4.85 & 4.85 & 2.74 & & 2.80 \\
5 & $\mathrm{HR}$ & 0.06 & 4.85 & 4.85 & 2.61 & 2.80 & 2.80 \\
6 & & 0.1 & 4.85 & 4.85 & 2.50 & & 2.80 \\
\hline
\end{tabular}

FZ: fault zone; HR: host rock.

thetic seismograms at the ground surface by doubling the amplitudes. This approximation is valid for "shear-wave window" area where the incident angle is less that the critical angle (about $35^{\circ}$ for the Poisson's ratio of 0.25) (Evans,
1984; Booth and Crampin, 1985).

In Nakamura and Takenaka (2005) only a pure strikeslip source with strike parallel to the fault zone was treated, and for this source type the formulation of the wave source was described. In this paper we also treat other source types. The formulation for any types of dislocation source is shown in Appendix B. We use the point dislocation with unit "potency" $\left(U_{0} d S ; U_{0}\right.$ : slip, $d S$ : surface element)(e.g., Takeuchi and Saito, 1972; Ben-Menahem, 1978) instead of unit seismic moment. Notice that the relation between the seismic moment and slip in anisotropic medium changes in variation of the slip direction, unlike the isotropic medium case, since the stiffness of the medium depends on the direction, and that the radiation pattern in anisotropy also is different from that of a double couple in isotropy (Kawasaki and Tanimoto, 1981; Vavryčuk, 2004). We use a parabolic pulse (Herrmann, 1979) with width of $0.25 \mathrm{~s}$ as a source time function and calculate synthetic seismograms with sampling frequency of $128 \mathrm{~Hz}$.

\section{Results}

Figure 2 shows the synthetic seismograms for the DS-B source for the anisotropic $(\varepsilon=0.06)$ and the isotropic $(\varepsilon=$ 0.00 ) fault zone. Figures $2(\mathrm{a})$ and (b) correspond to the 
$\varepsilon=0.0$

source : inside FZ $\varepsilon=0.02$

source : inside FZ $\varepsilon=0.06$

source : inside FZ $\varepsilon=0.1$

source : inside FZ
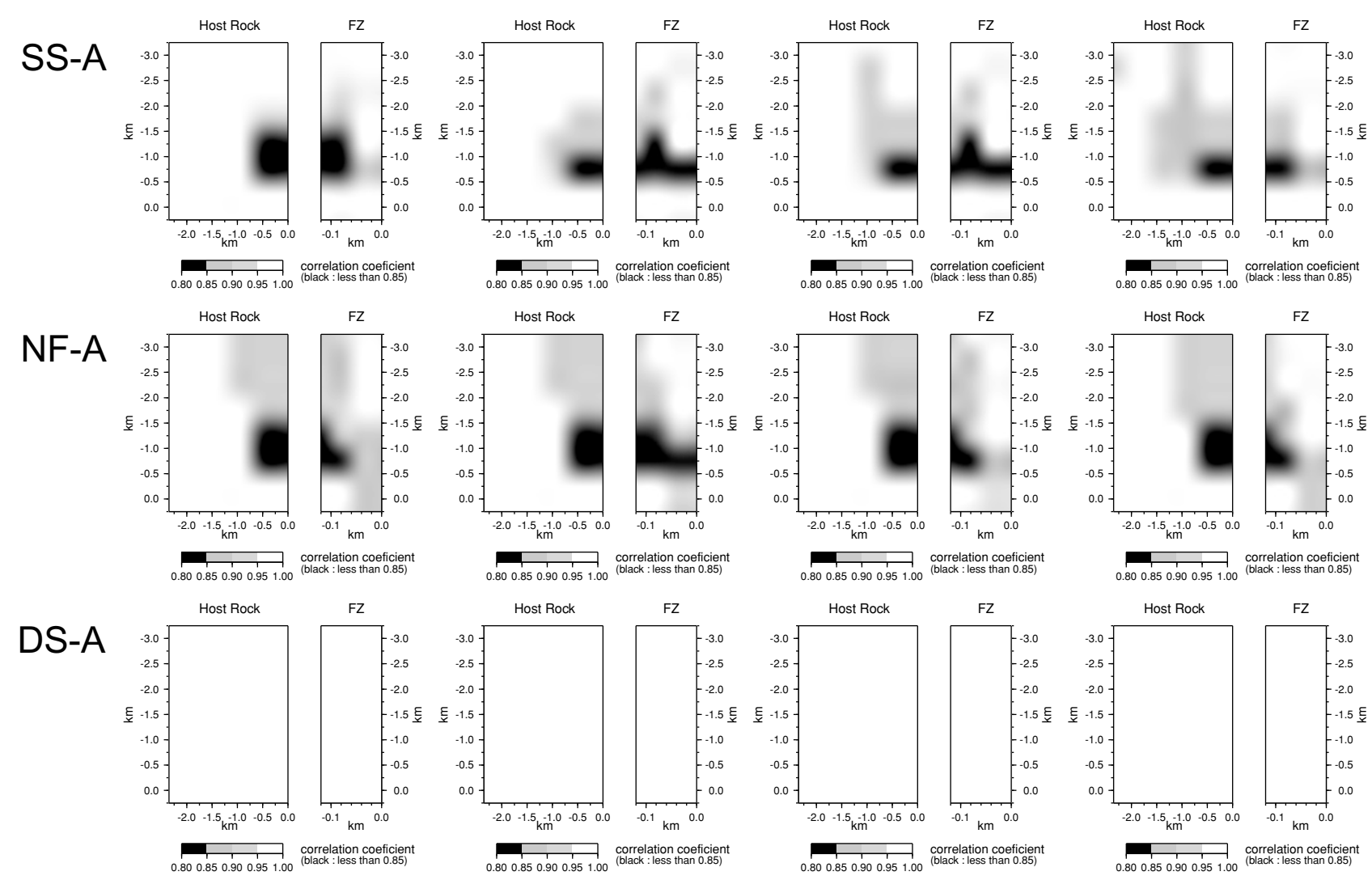

SS-B
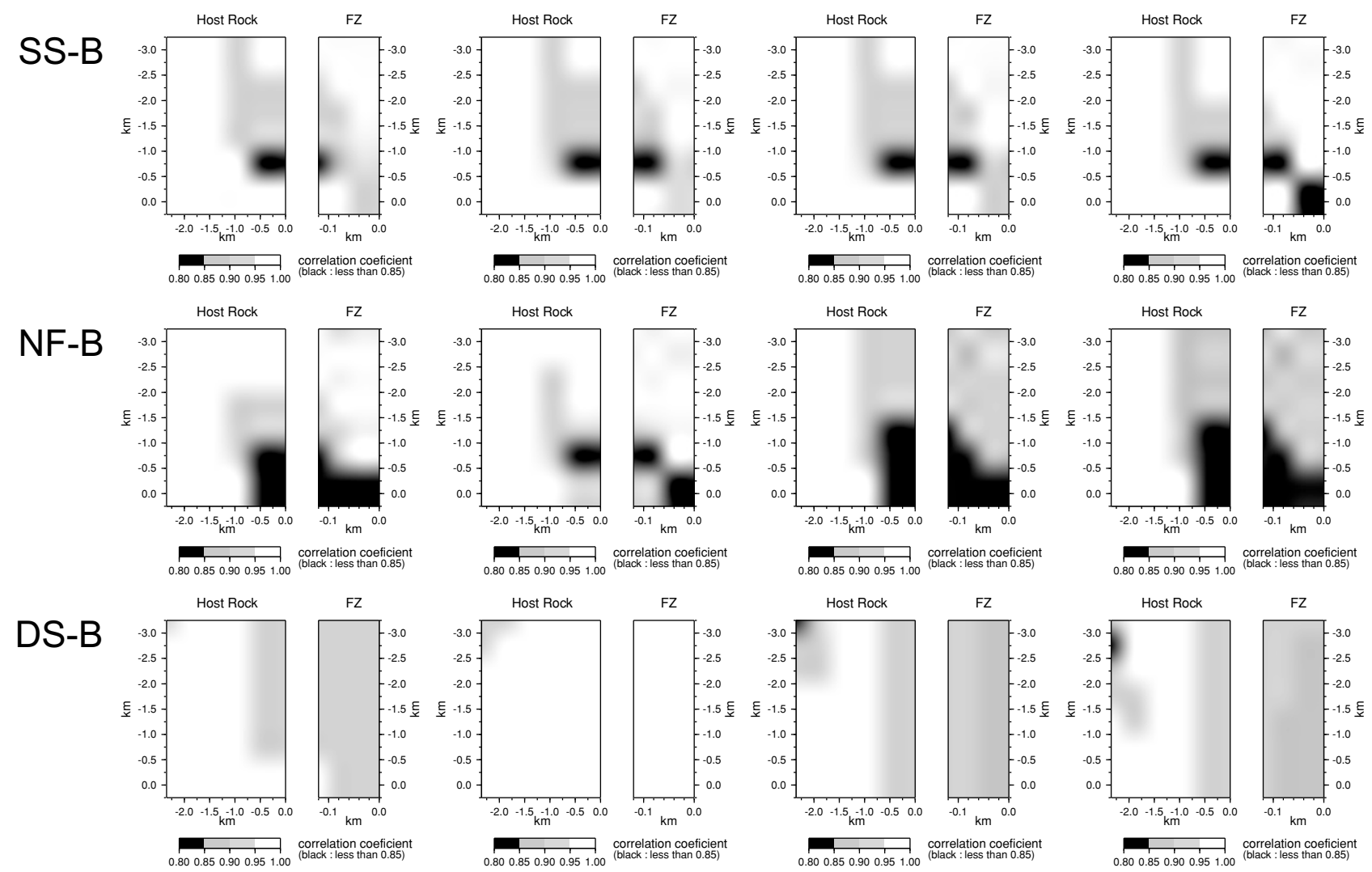

Fig. 3. Cross-correlation coefficient with the cross-correlation method at station in the fault zone area and the host rock area (outside the fault zone) in the case of the source located inside the fault zone. The panels from the left show the cases of crack density 0.0 (isotropy), $0.02,0.06$ and 0.1 , respectively. The panels from the top show the cases of SS-A, NF-A, DS-A, SS-B, NF-B and DS-B source, respectively. Black colored area indicates the coefficients less than 0.85 . 


$$
\varepsilon=0.0
$$

source : inside $\mathrm{FZ}$

$$
\varepsilon=0.02
$$

source : inside FZ
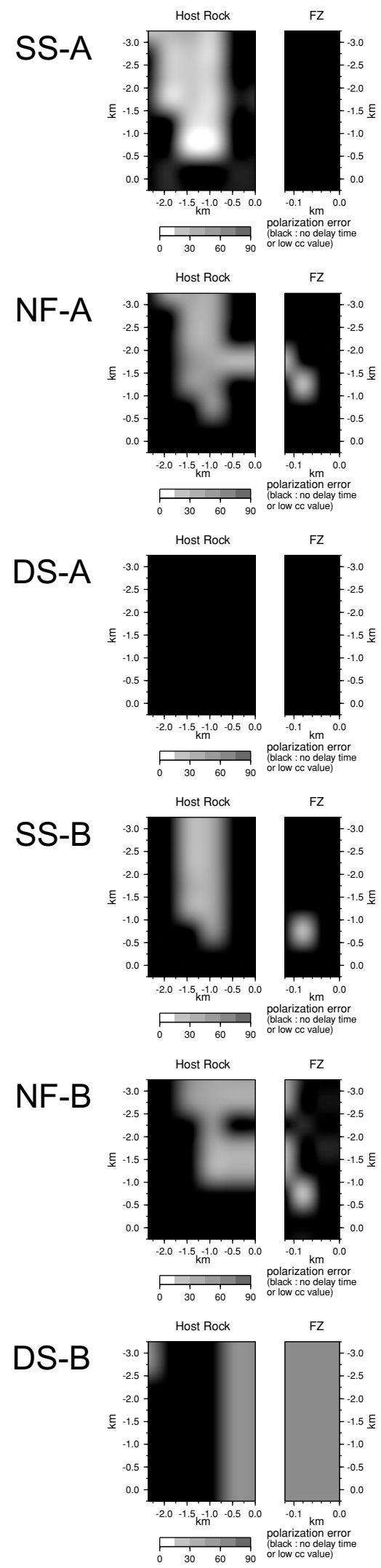
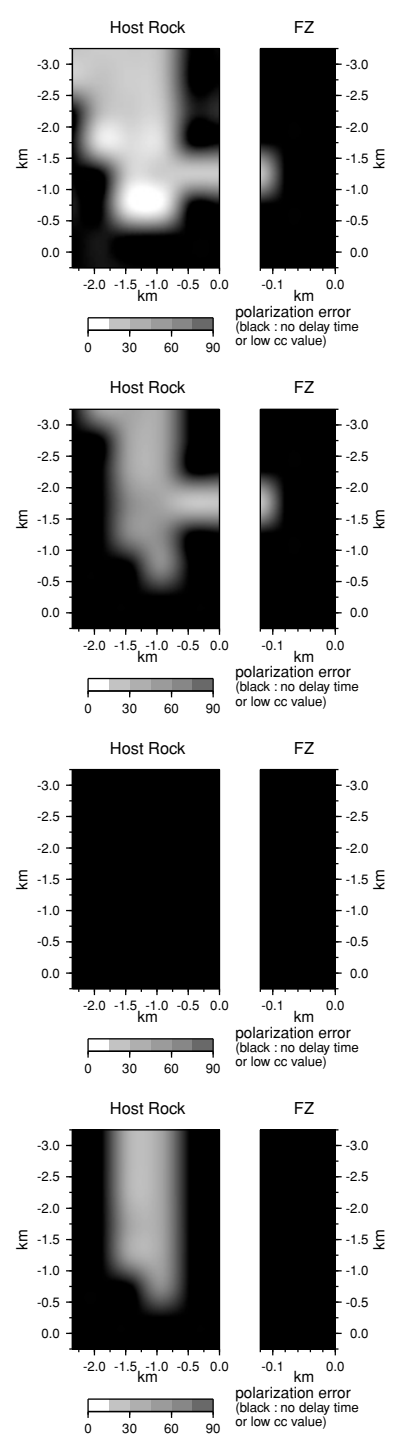

Host Rock
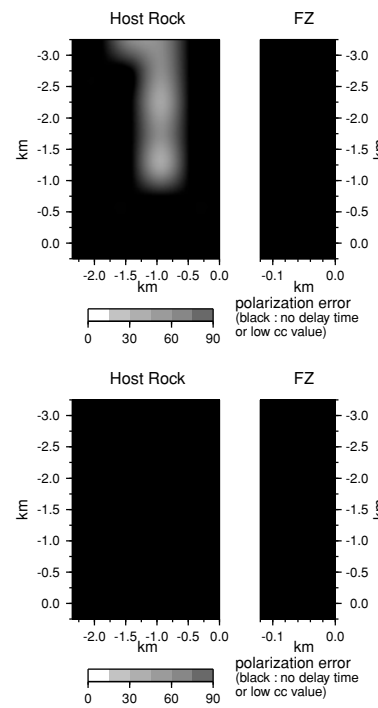

$\varepsilon=0.06$

source : inside FZ

$\varepsilon=0.1$

source : inside FZ
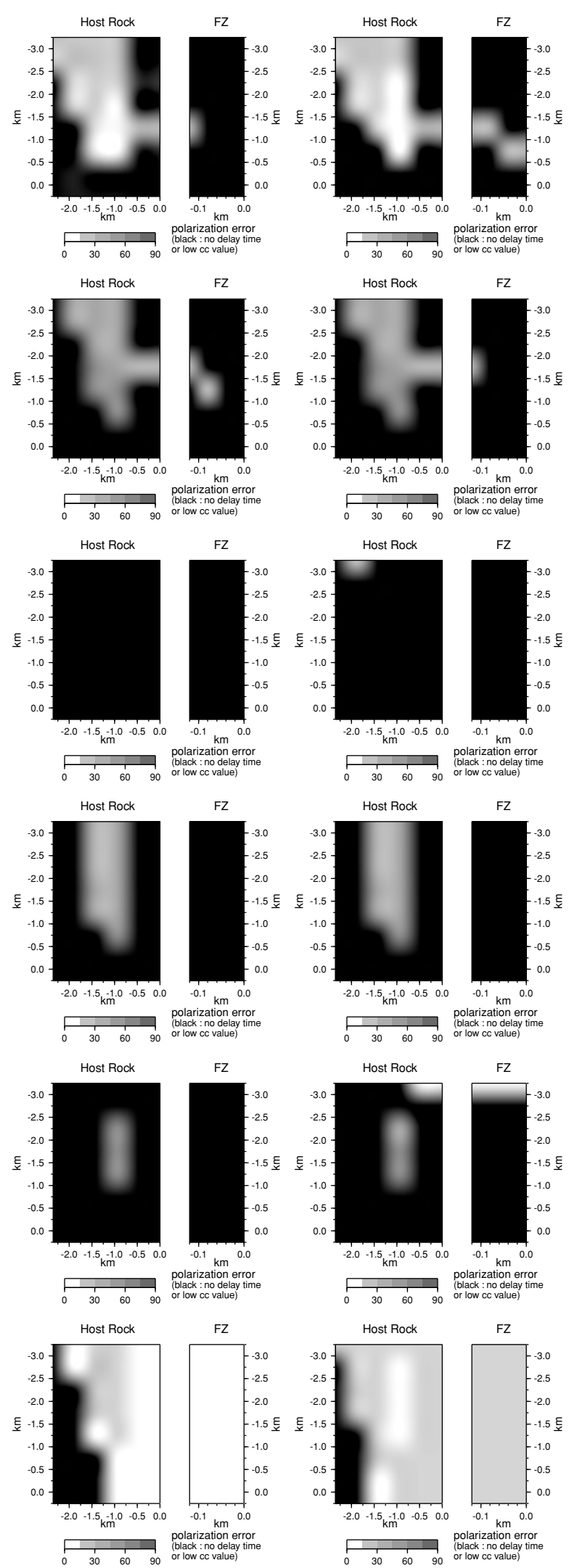

Fig. 4. Residual between the polarization direction of $q S_{1}$-wave measured with the cross-correlation method and the true direction (i.e. the $x_{2}$ direction) in the case of the source located inside the fault zone. Black colored area indicates the coefficients less than 0.85 or delay time less than $0.0078125 \mathrm{~s}$ (one time sampling point). 


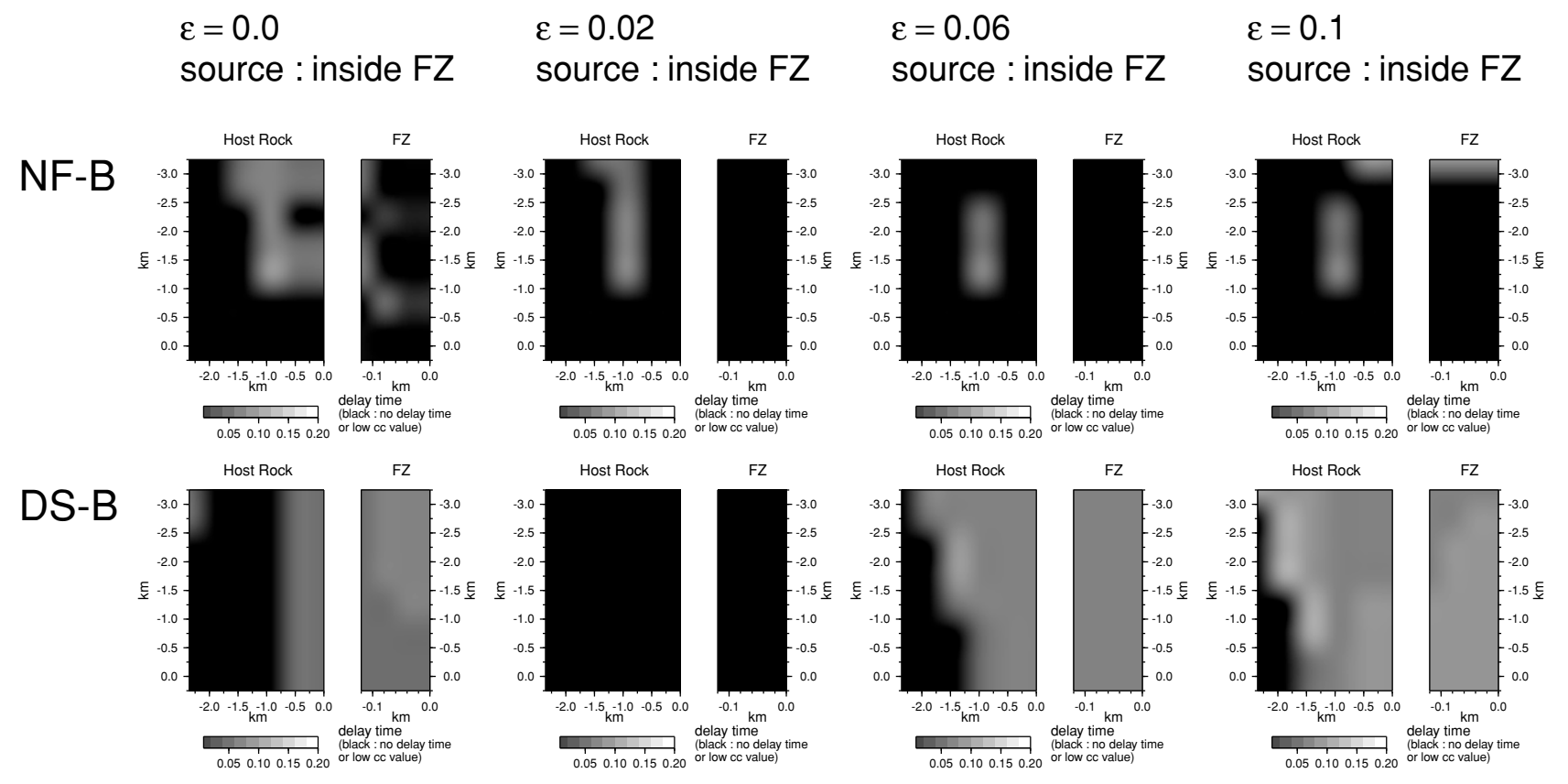

Fig. 5. Measured delay time between arrivals of $q S_{1}$ - and $q S_{2}$ wave for NF-A and DS-B sources located inside the fault zone. Black colored area indicates the coefficients less than 0.85 or delay time less than $0.0078125 \mathrm{~s}$ (one time sampling point).

cases of the source located inside (open star in Fig. 2(d)) and outside the fault zone (solid star in Fig. 2(d)), respectively. The synthetic seismograms for the other source types are shown in Fig. C1 in Appendix C. The station name used here is shown in Fig. 2(d). Note that in the anisotropic fault zone model we studied here, $q S_{1}$-wave has a polarization on the $x_{1}-x_{2}$ plane, while $q S_{2}$-wave has the polarization parallel to the $x_{3}$ axis (Fig. 2(d)).

For DS-A and SS-A sources located inside the fault zone, the amplitude in $x_{2}$ component is almost zero or small at stations A1-3 and B1-7, while for DS-B source almost the same as that of $x_{3}$ component due to its source mechanism (Fig. C1). For NF-A, SS-B and NF-B sources, the amplitude in $x_{2}$ component is dominant (Fig. $\mathrm{C} 1$ ). We identify the longer pulse width of the shear-wave portion (around 1.8 to $3.0 \mathrm{~s}$ ) in the $x_{3}$ component of the seismograms in the anisotropic fault zone for all sources than those in the isotropic fault zone (Figs. 2(a) and C1). For all sources outside the fault zone, although the shear wave portion in the case of the anisotropic fault zone is very slightly longer than that in isotropic case, the difference between the waveforms for both cases is too small to be detected. Because the propagation distance in the fault zone is so short compared with the whole path length, it does not affect waveforms significantly. These differences of source types and source locations cause the estimation errors in parameters of anisotropy. We will discuss this issue in the next section.

In Figure 2(a), at stations $\mathrm{A} 1-\mathrm{A} 3$ and $\mathrm{B} 1-\mathrm{B} 7$ near the fault zone, the seismograms in the $x_{3}$ component have complex waveforms compared with those at A4 and A5. Their complexities disappear at stations away from the fault zone or for the source located outside the fault zone. We see a later phase arriving a few hundreds of milliseconds after the main shear-wave in the anisotropic seismograms at these stations A1-A3 and B1-B7 (upper traces in Fig. 2(c)). This phase is clearly seen for stations far from the source along the strike of the fault zone, for example at stations B6 and B7. At all stations in isotropic seismograms, this phase cannot be seen (lower traces in Fig. 2(c)).

The first arriving shear-wave is the head wave propagating between the fault zone and the host rock, followed by the direct $q S_{1}$-wave ( $x_{2}$-component) and direct $q S_{2}$-wave ( $x_{3}$-component). The later phase seen on the $x_{3}$-component synthetics for the anisotropic fault zone consists of this direct $q S_{2}$-wave and its reverberation. The $q S_{2}$-wave is the slower split shear-wave with polarization direction parallel to the symmetry axis $x_{3}$. The later phase appears only in the $x_{3}$ component for the anisotropic fault zone, from the arrival of direct $q S_{2}$-wave expected by Hudson's (1980, 1981) model. The later phase is seen clearly in the case of higher crack density $\varepsilon$ and long propagation distance inside the fault zone. At stations away from the fault zone (stations A4 and A5 in Fig. 2(a)), this phase appears obscurely, or is not seen at all. As the crack density increases, the velocity of $q S_{2}$-wave inside the fault zone becomes lower, and the velocity contrast between the fault zone and the host rock becomes higher, which amplifies the reverberation. In Hudson's $(1980,1981)$ crack model, the velocity of $q S_{1}$-wave is almost independent of the crack density for the incidence nearly perpendicular to the symmetry axis of anisotropy. At stations inside the fault zone, this incidence is almost perpendicular to the $x_{3}$-axis (symmetry axis) and the velocity of $q S_{1}$-wave is almost the same as that of isotropy, so that the reverberation of $q S_{1}$-wave can little be amplified (Fig. 2(c)). The most energy of the reverberation phase is trapped inside the fault zone, and this phase is not seen at stations for the source located outside the fault zone (Fig. 2(b)), since the propagation distance in the fault zone is too short. These characteristics of the $q S_{2}$ reverberation phase may help us catch the position of the cracked 
region in the fault zone and source location if we observe this phase associated with the $q S_{2}$-wave propagation.

\section{Discussion}

The shear-wave splitting is described by two parameters: the polarization direction $\phi$ of $q S_{1}$-wave and the delay time $\delta t$ between the arrivals of $q S_{1}$ - and $q S_{2}$-wave. We here try to measure these parameters from the synthetic seismograms with the cross-correlation method (Bowman and Ando, 1987) in order to investigate which source type and station location would be more appropriate to estimate $\phi$ and $\delta t$ correctly. The cross-correlation method gives $\phi$ and $\delta t$ when the cross-correlation coefficient for two components of seismogram is maximum, after coordinate transform and time shift for the components. In most of the recent observational studies on anisotropy this method or similar methods have been employed.

We use the time window with $0.5 \mathrm{~s}$ width to the first portion of shear-wave in the $x_{2}$ and $x_{3}$ components. Figure 3 show the values of the cross-correlation coefficient at the stations for the source located inside the fault zone. For most cases the correlation coefficient is around 1.0 except some stations near the fault zone, which implies the feasibility of determining the splitting parameters except near the fault zone. At stations near the fault zone in the case of SSA and -B and NF-A and -B source, estimation of splitting parameters from the seismogram is not succeeded because of the cross-correlation coefficient value less than 0.85 , as shown by black colored area in Fig. 3. It is due to the presence of the waves generated by the interaction of the direct waves with the fault zone boundaries, such as the head wave and the reverberation phases. For DS-A source, the coefficient is high even at stations near the fault zone. However it is meaningless because the $x_{2}$ component has almost no energy (Fig. C1). For the source outside the fault zone (Fig. D1), since the shear-waves pass too short length in the fault zone to be affected by the medium of the fault zone, all stations show high cross-correlation coefficient values.

Figure 4 shows the residuals between the correct polarization ( $x_{2}$ direction) angle and the obtained $\phi$ for the source located inside the fault zone. The stations with the crosscorrelation value less than 0.85 or the delay time $\delta t$ less than $0.0078125 \mathrm{~s}$ corresponding to one time sampling point are represented by black colored area, which indicates low reliability for constraining the splitting parameters. Since we take the symmetry axis of anisotropy in the $x_{3}$-axis, the direction $\phi$ due to anisotropy should be parallel to the $x_{2}$ direction, perpendicular to the symmetry axis, that is $\phi=0^{\circ}$ or $180^{\circ}$ measured from the $x_{2}$-axis. Although most cases have large residuals with more than $15^{\circ}$ (dark colored area in Figs. 4 and D2), we can extract almost correct values of $\phi$ at some stations near the fault zone for NF-B and DS-B sources (white colored area in Figs. 4 and D2) and NF-A source (Fig. D2). For SS-A (Fig. 4) and SS-B (Fig. D2), the small residuals indicated by white are also seen, but found even in $\varepsilon=0.0$ (isotropy). These small residuals for sources SS-A and SS-B may be a ghost which generated by the effects of the structure, as Nakamura and Takenaka (2005) suggested for SS-A source. The distortion of shear-wave due to the effects of the structure may result in small resid- uals even for $\varepsilon=0.0$.

Figures 5 and D3 (Appendix D) show the obtained $\delta t$ at stations for NF-B and DS-B sources located inside the fault zone and NF-A, NF-B and DS-B sources outside the fault zone, respectively, where $\phi$ with the small residuals are available. The theoretical $\delta t$ calculated from Hudson's $(1980,1981)$ model is $0.05-0.33 \mathrm{~s}$ and $0.0002-0.001 \mathrm{~s}$ for crack densities $\varepsilon=0.02-0.1$ at the stations inside and outside the fault zone, respectively. Obtained $\delta t$ at some stations do not show good agreements with the theoretical $\delta t$. This is mainly that at stations outside the fault zone the propagation distance in anisotropy is too short and at stations inside the fault zone the velocity structure affects the waveform. Although the cross-correlation method might have little resolution for retrieving the correct $\delta t$ in the fault zone, the results for NF-A, NF-B and DS-B sources show significant differences between the case of the isotropic and anisotropic fault zone and provides an informative clue for demonstrating the existence of the anisotropic fault zone. For the reverse fault, the same results as those of NF-A and -B are obtained.

For the 1995 Hyogo-ken Nanbu earthquake, about 17\% of the aftershocks have source mechanisms different from the regional stress field, which are called "atypical" aftershocks (Yamanaka et al., 2002). In our case, the source mechanisms except SS-A may correspond to atypical events. Our results show that analyzing such atypical events, especially NF-A, NF-B and DS-B sources, are available for detecting the anisotropic fault zone although it is difficult to estimate it from the records for SS-A source. Thus, we suggest that the relative position of recording stations to the fault zone, highly accurate source location and use of "atypical" events are needed for reliable estimation of the splitting parameters.

The main problem of measuring shear-wave splitting with the cross-correlation and some other methods such as the linearity techniques: use of the aspect-ratio of horizontal particle motion (e.g., Shih et al., 1989) is that they assume a homogeneous anisotropic medium. This assumption is often invalid in seismic observations near the fault zone. The fault zone usually has a strong velocity reduction as high as $10-50 \%$ on shear-wave velocity relative to the host rock (e.g., Li et al., 1990, 1997; Hough et al., 1994), and the effects depend on the source type significantly. Therefore, analyzing the seismograms, we need to remove these effects or take into account them. Our study suggests the necessity of the appropriate combinations of station locations and source type to extract shear-wave splitting even for a simple fault zone structure. In order to estimate parameters of anisotropy correctly for more complex structure and any source types, we may need to use synthetic seismograms for more realistic structure models.

\section{Conclusion}

In order to quantify the effects of anisotropy in a fault zone, we calculated synthetic seismograms in a fault zone model represented by a vertically layered medium with flat interfaces, in which one layer corresponding to the fault zone is anisotropic. We assumed several types of seismic wave sources. The synthetic seismograms show the 


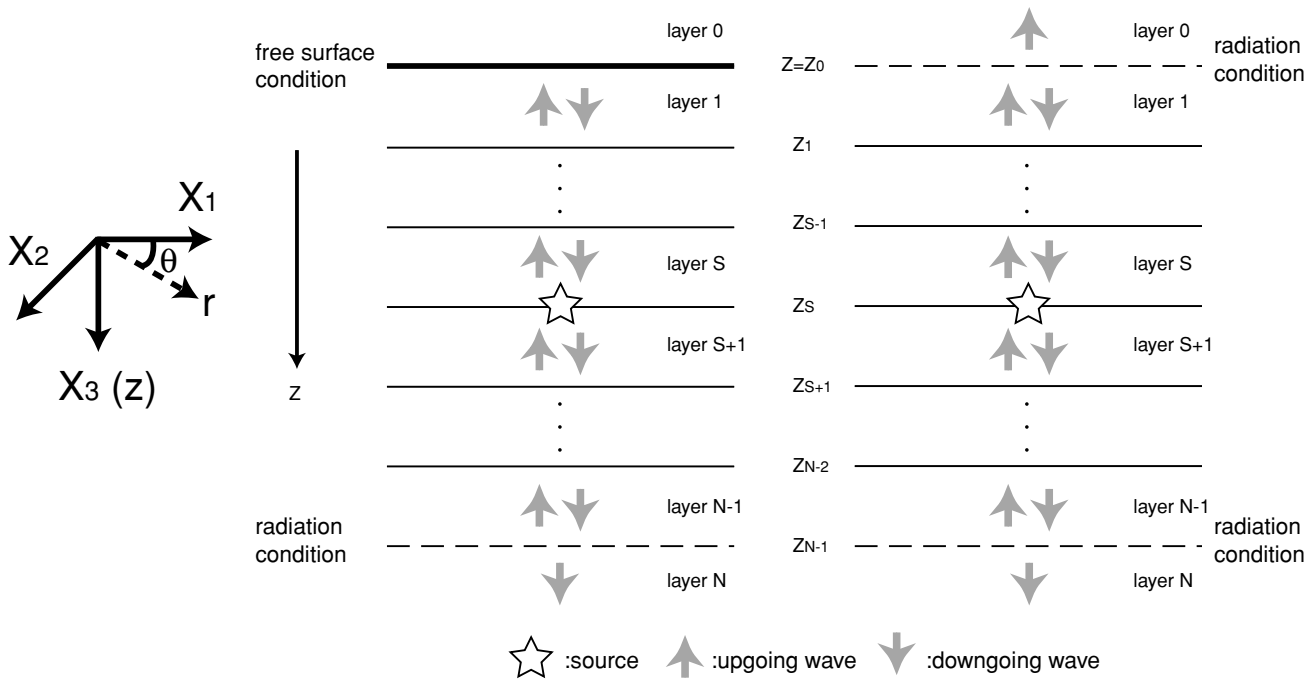

Fig. A1. Schematic illustration of upgoing and downgoing waves in the case of free surface condition (left) or radiation boundary conditions (right) set at level $z=z_{0}$. All lines are the layer boundaries. The free surface and radiation conditions are applied to the planes indicated by thick and broken lines, respectively.
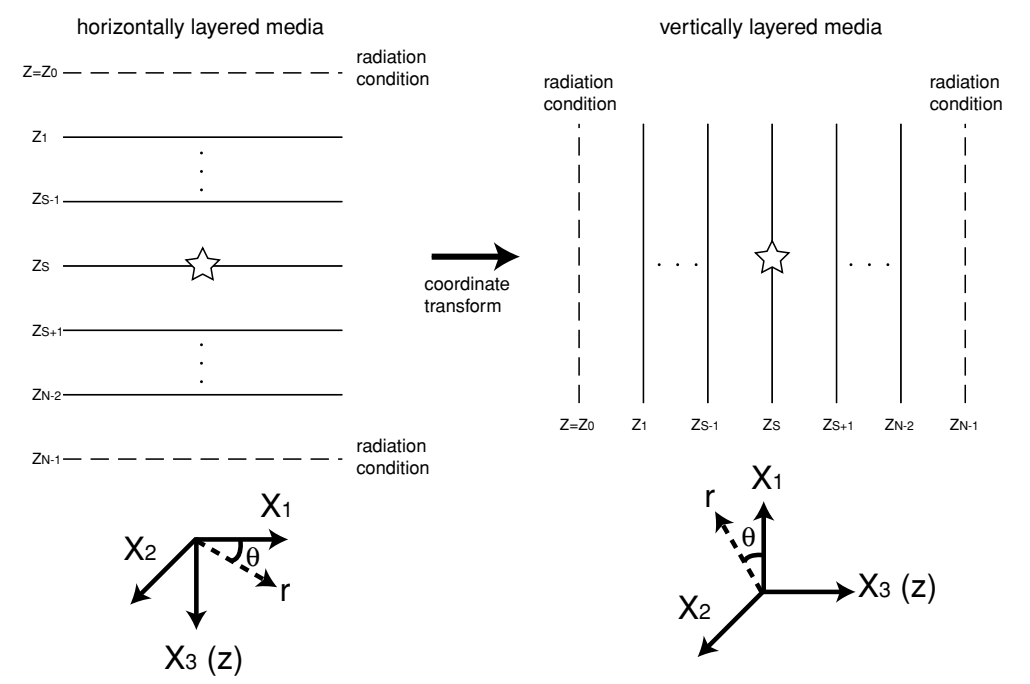

Fig. A2. Schematic illustration of the coordinate transform for calculation of seismic waves in a vertically layered medium.

arrival of a later phase after the first arriving shear-wave, at stations along and across the fault zone. We found that the time delay between the shear-wave and the later shearwave phase is proportional to the propagation distance in the anisotropic fault zone and to the degree of anisotropy. If we deploy seismic stations around and along the main fault, calculating these time delay could provide a clue to detect the fault zone. We also found that, in the case of a pure strike slip source, the seismic waves at stations near the fault zone are more affected by the velocity structure than by the anisotropy of the fault zone. In this case, from usual analysis such as the cross-correlation method we may not be able to detect the anisotropic fault zone and not estimate the correct shear-wave splitting parameters. However, the anisotropic fault zone can be detected, when the fault zone has large crack density, for normal-fault and dipslip sources located outside the fault zone with the strike of $45^{\circ}$ or parallel to the fault zone, and for normal-fault and dip-slip sources inside the fault zone with the strike of $45^{\circ}$ against the fault zone. It suggests that the information on the mechanism and location of the events relative to the fault zone are needed to estimate anisotropic properties in the fault zone.

Acknowledgments. We thank Prof. Kin'ya Nishigami and Dr. Yutaka Mamada for useful discussions. We appreciate the constructive comments of Editor Dr. Eiichi Fukuyama, reviewer Dr. Enru Liu and an anonymous reviewer. GMT (Wessel and Smith, 1991) was used for making several figures. Mr. Jafar Gandomi Arash kindly read the manuscript. This study was partially supported by the fund of Disaster Prevention Research Institute, Kyoto University (Research No. 16G-02).

\section{Appendix A. The Displacement-stress Vector on the Radiation Boundary Condition}

We define the displacement-stress vector $\boldsymbol{b}(z)$ using the displacement $u_{i}$ and the stress component $\sigma_{i j}$ in the frequency domain under the expansion of the surface vector harmonics in the cylindrical coordinates $(r, \theta, z)$ as

$$
\boldsymbol{b}(z)=\left[u_{r}, u_{z}, \sigma_{z z}, \sigma_{r z}\right]^{T}(P-S V), \boldsymbol{b}(z)=\left[u_{\theta}, \sigma_{\theta z}\right]^{T}(S H) .
$$




\section{source inside the fault zone}

SS-A
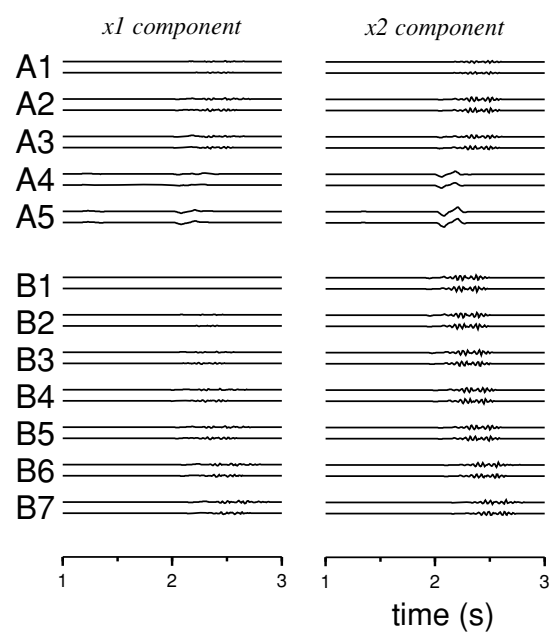

\section{NF-A}
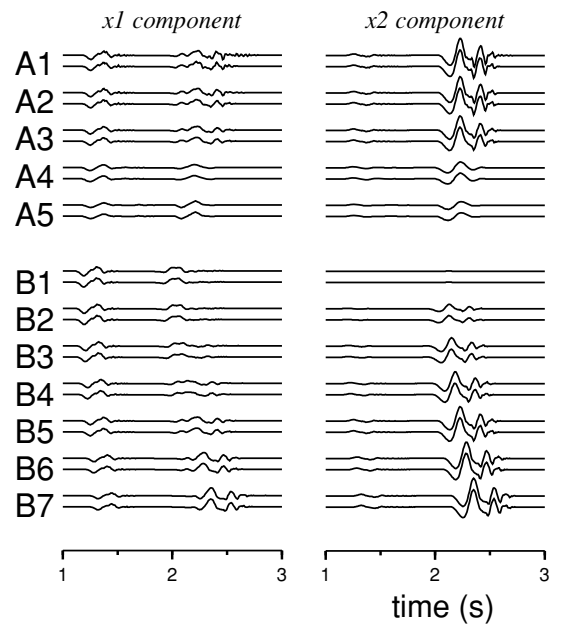

\section{DS-A}

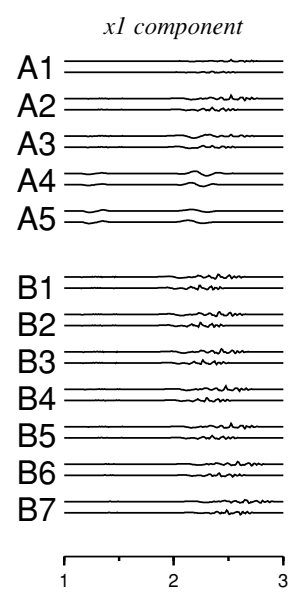

\section{source outside the fault zone}

SS-A
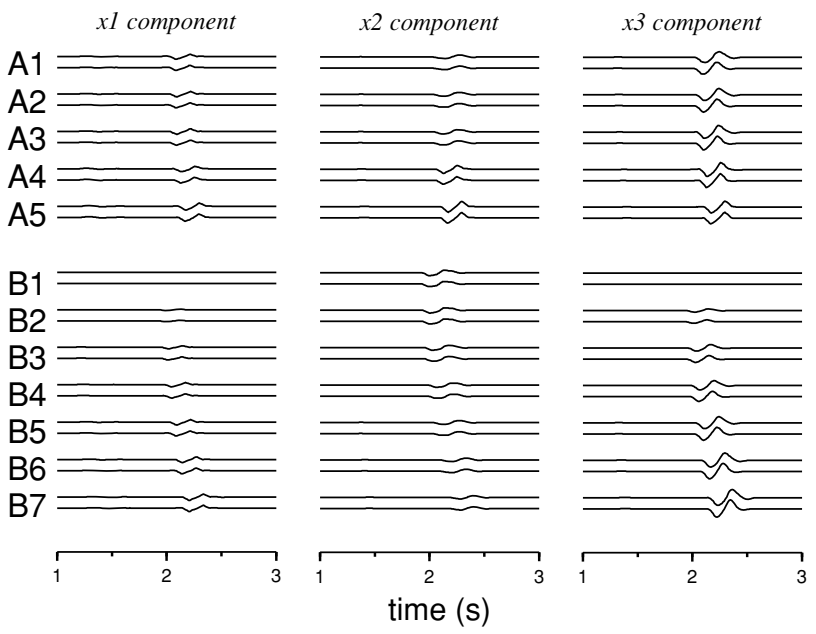

NF-A

x3 component
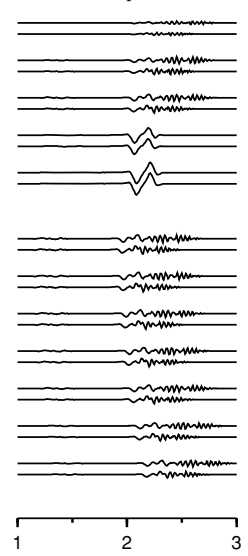

$x 1$ component

$\mathrm{A} 1 \approx \approx \approx$

$\mathrm{A} 2 \approx$

$\mathrm{A} 3 \approx \approx$

$\mathrm{A} 4 \approx$

$\mathrm{A} 5 \approx$

$\mathrm{B1} \approx \approx$

$\mathrm{B} 2 \approx$

$\mathrm{B} 3 \approx$

$\mathrm{B} 5 \approx$

$\mathrm{B} 6 \approx$

$\mathrm{B} 7 \approx$

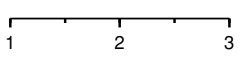

$x 2$ component

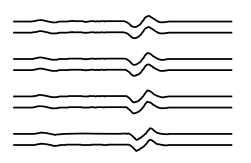

=

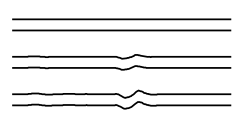

$\approx$

$\approx$

$\approx \approx$

$\approx \approx$

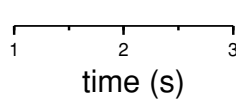

x3 component
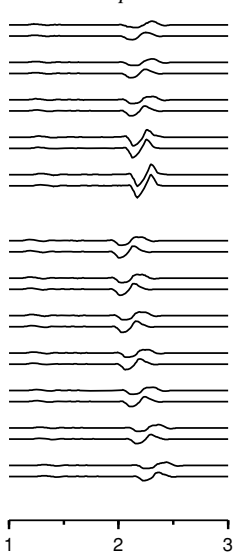

time (s)

\section{DS-A}

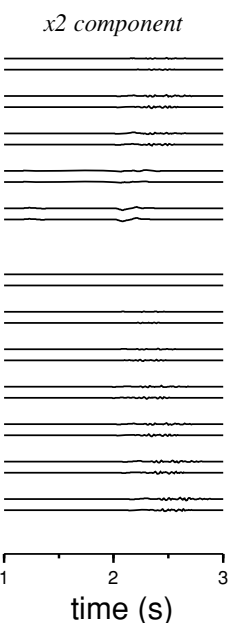

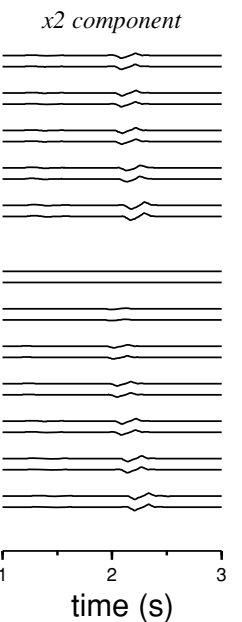

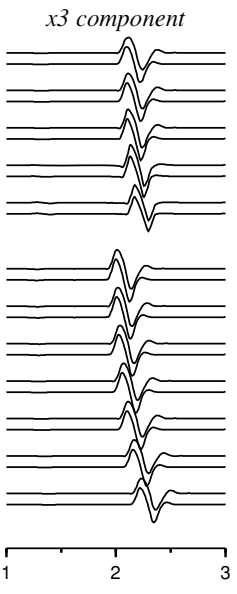

Fig. C1. Synthetic seismograms in the case of SS-A, -B, NF-A, -B, and DS-A. Left and right panels show synthetic seismograms for the source located inside and outside the fault zone, respectively. Upper and lower traces at each station name show the seismogram through the anisotropic $(\varepsilon=0.06)$ and isotropic $(\varepsilon=0.0)$ fault zone, respectively. The location of station is shown in Fig. 2. 


\section{source inside the fault zone}

\section{SS-B}

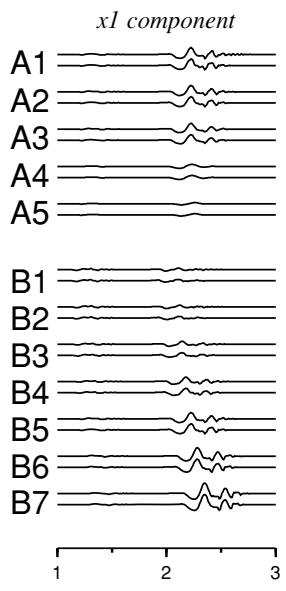

NF-B
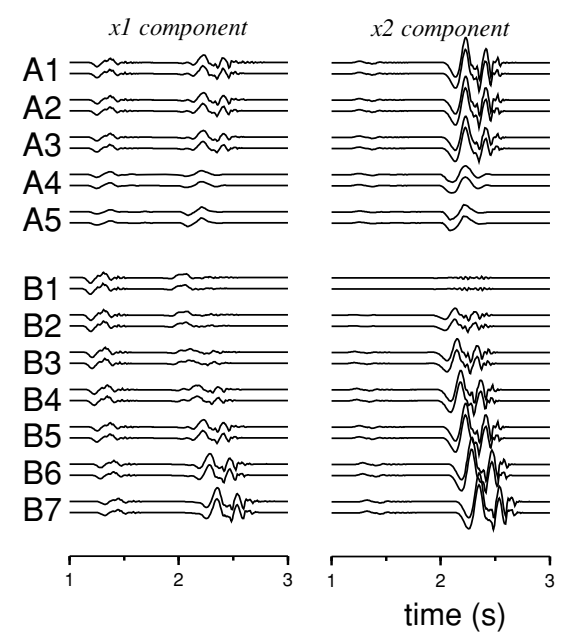

source outside the fault zone

\section{SS-B}
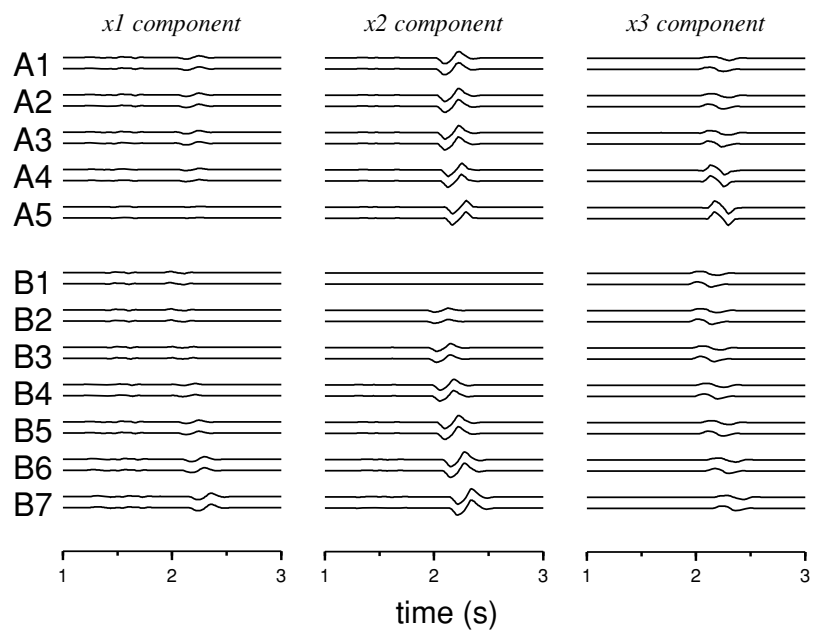

NF-B

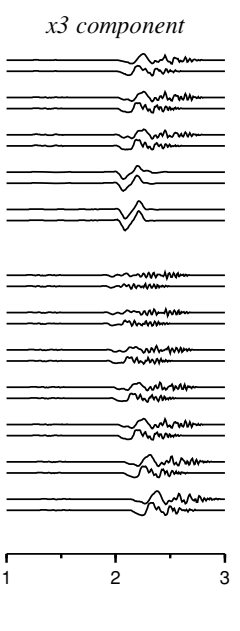

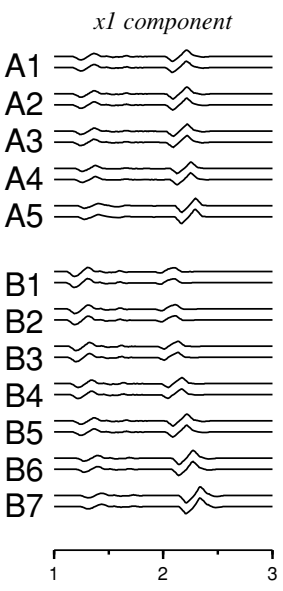

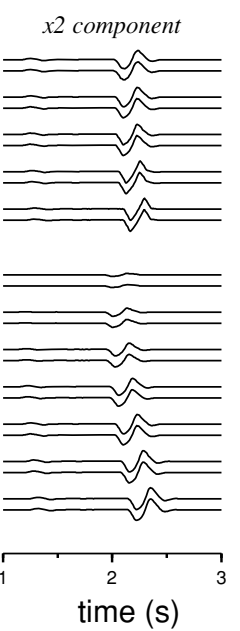

Fig. C1. (continued).

(A.1) $\boldsymbol{S}$ is the source vector (Appendix B). $\boldsymbol{P}_{N}$ is the propagator matrix in layer $N$ defined by,

$$
\boldsymbol{P}_{N}=\boldsymbol{D}_{N} \boldsymbol{E}\left(z_{N}-z_{N-1}\right) \boldsymbol{D}_{N}^{-1} .
$$
vector harmonics is
equations using $\boldsymbol{b}(z)$,

$$
\frac{d \boldsymbol{b}(z)}{d z}=\boldsymbol{A} \boldsymbol{b}(z),
$$

where $\boldsymbol{A}$ is a $4 \times 4(P-S V)$ or $2 \times 2(S H)$ matrix whose elements represent material properties. The relation between $\boldsymbol{b}\left(z_{0}\right)$ and the constant vector $\boldsymbol{K}_{N}$ in layer $N$ which consists of coefficients of upgoing and downgoing waves are established (e.g., Wang and Herrmann, 1980) as,

$$
\boldsymbol{K}_{N}=\boldsymbol{X} \boldsymbol{S}+\boldsymbol{R} \boldsymbol{b}\left(z_{0}\right),
$$

where

$$
\begin{gathered}
\boldsymbol{X}=\boldsymbol{D}_{N}^{-1} \boldsymbol{P}_{N-1} \cdots \boldsymbol{P}_{S+1}, \\
\boldsymbol{R}=\boldsymbol{D}_{N}^{-1} \boldsymbol{P}_{N-1} \cdots \boldsymbol{P}_{1} .
\end{gathered}
$$

$\boldsymbol{D}_{N}$ is the eigenvector matrix of $\boldsymbol{A}$ in layer $N$, which transforms the vector of coefficients of upgoing and downgoing waves into the displacement-stress vector, and $\boldsymbol{E}_{N}$ is the diagonal matrix which explains the phase shift from $z_{N-1}$ to $z_{N}$.

Imposing the free surface condition and the radiation boundary condition at $z=z_{0}$ and $z_{N-1}$, respectively (Fig. A1), we can derive the displacement-stress vector at $z_{0}$ from Eq. (A.3) in the following form:

$$
\begin{aligned}
& \boldsymbol{b}\left(z_{0}\right)=\frac{1}{R_{11} R_{22}-R_{12} R_{21}}\left[\begin{array}{c}
\left(R_{12} X_{2 i}-R_{22} X_{1 i}\right) S_{i} \\
\left(R_{21} X_{1 i} S_{i}-R_{11} X_{2 i}\right) S_{i} \\
0 \\
0
\end{array}\right] \\
& i=1,2,3,4 . \quad(P-S V) \text {; }
\end{aligned}
$$




\section{$\varepsilon=0.0$ \\ source : outside FZ}

$\varepsilon=0.02$

source : outside FZ $\varepsilon=0.06$

source : outside FZ $\varepsilon=0.1$

source : outside FZ
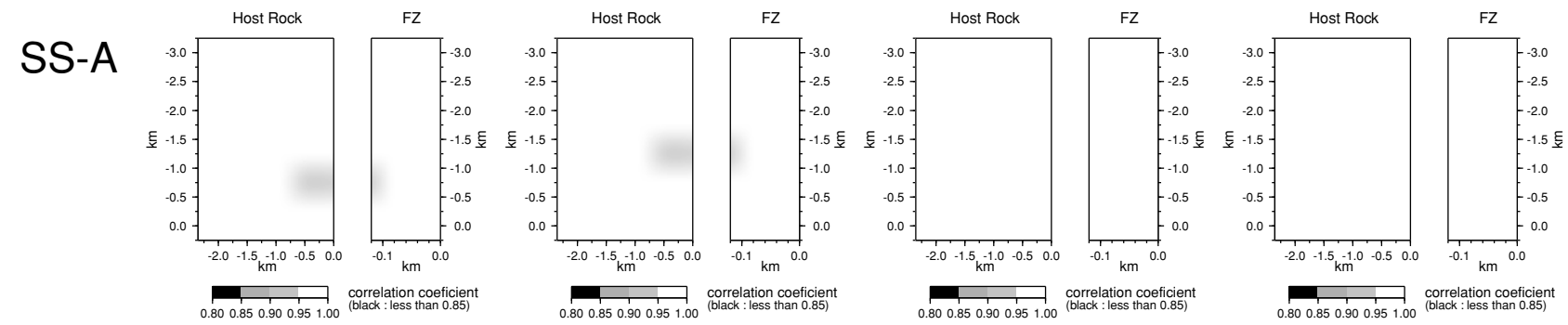

NF-A
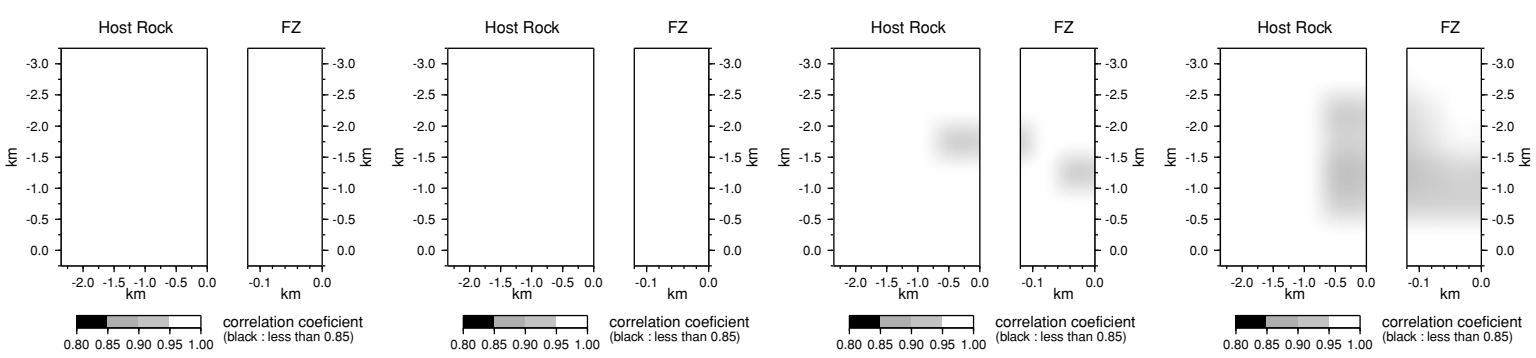

$\underbrace{0.85}_{0.80} 0.900 .951 .00$ (black less than 0.85 )
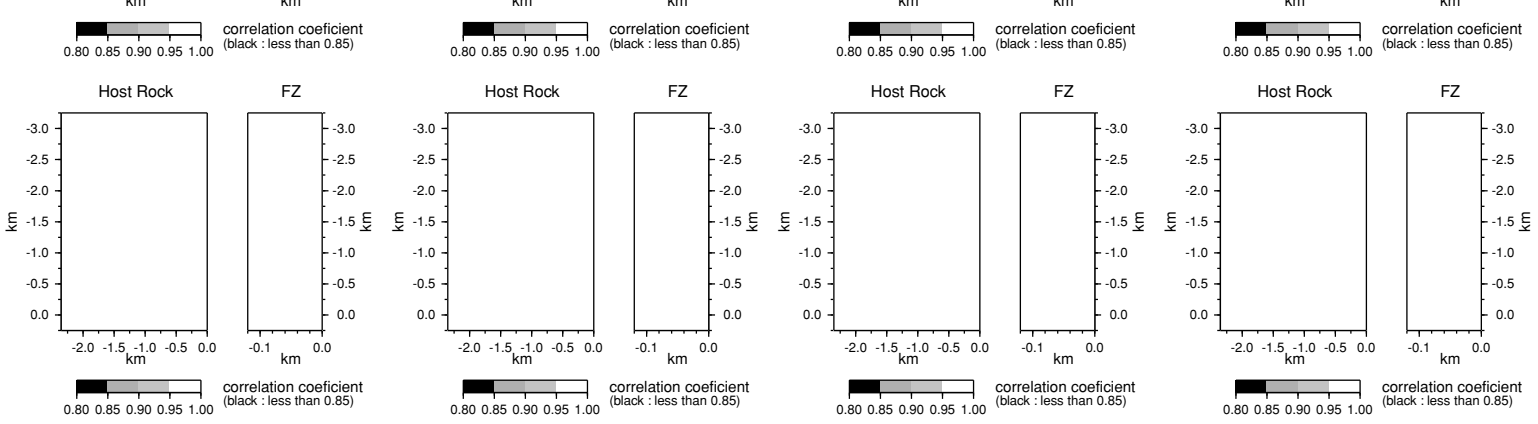

SS-B
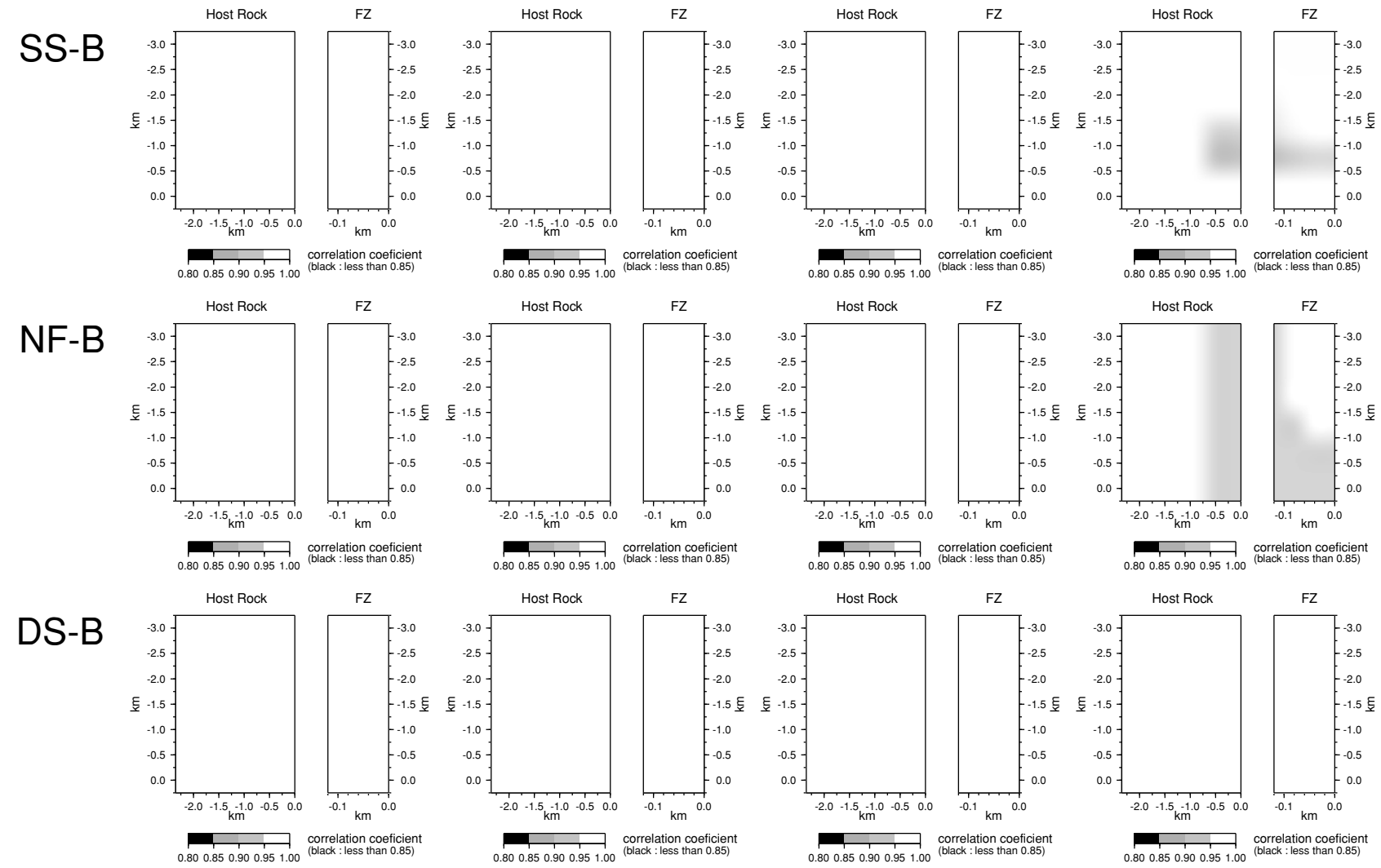

Fig. D1. Cross-correlation coefficient with the cross-correlation method at station in the fault zone area and the host rock area (outside the fault zone) in the case of the source located outside the fault zone. Other notations are the same as in Fig. 3. 


$$
\begin{array}{llll}
\varepsilon=0.0 & \varepsilon=0.02 & \varepsilon=0.06 & \varepsilon=0.1 \\
\text { source : outside FZ } & \text { source : outside FZ } & \text { source : outside FZ } & \text { source : outside FZ }
\end{array}
$$
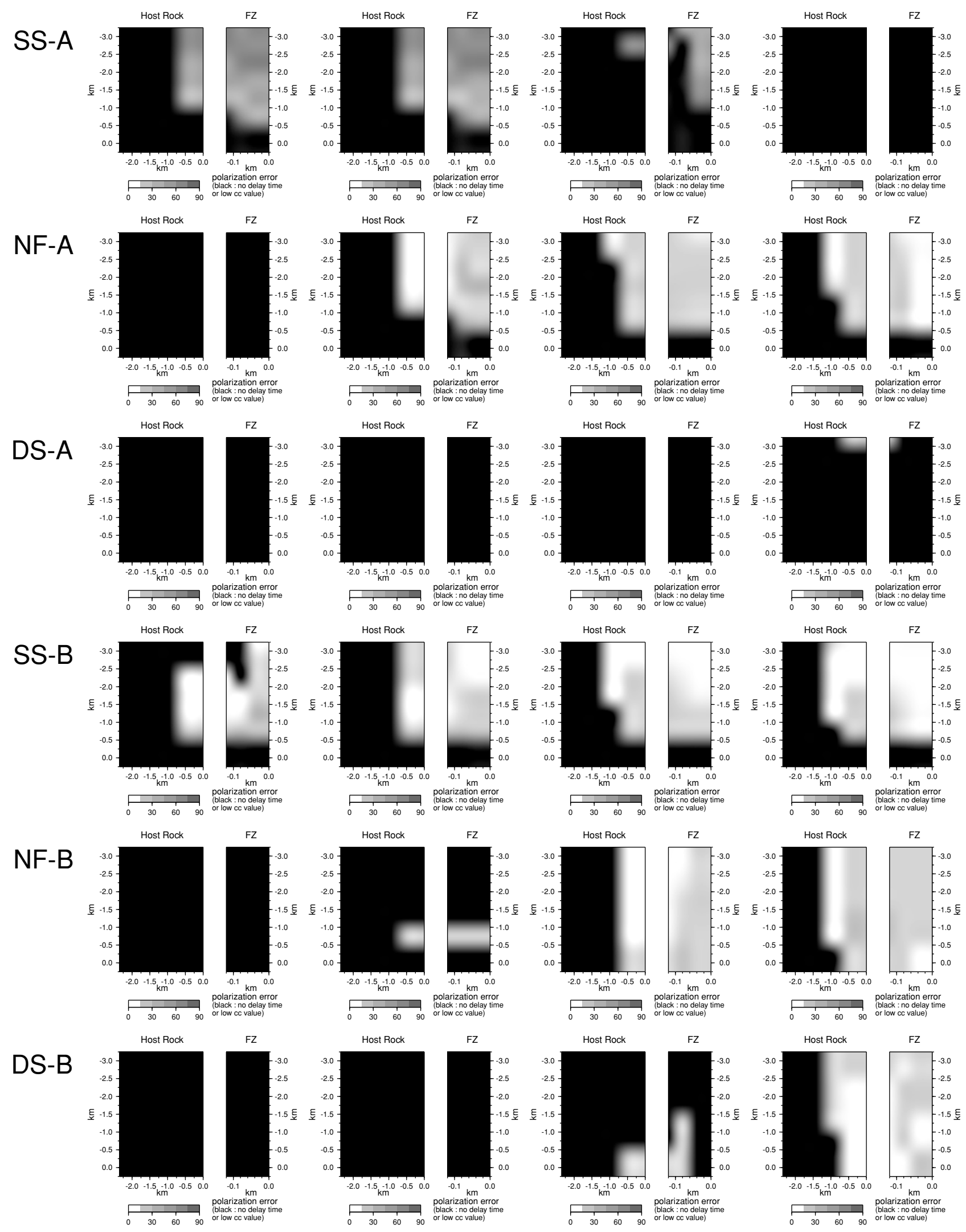

Fig. D2. Residual between the polarization direction of $q S_{1}$-wave measured with the cross-correlation method and the true direction (i.e. the $x_{2}$ direction) in the case of the source located outside the fault zone. Other notations are the same as in Fig. 4. 


$$
\begin{array}{llll}
\varepsilon=0.0 & \varepsilon=0.02 & \varepsilon=0.06 & \varepsilon=0.1 \\
\text { source : inside FZ } & \text { source : inside FZ } & \text { source : inside FZ } & \text { source : inside FZ }
\end{array}
$$
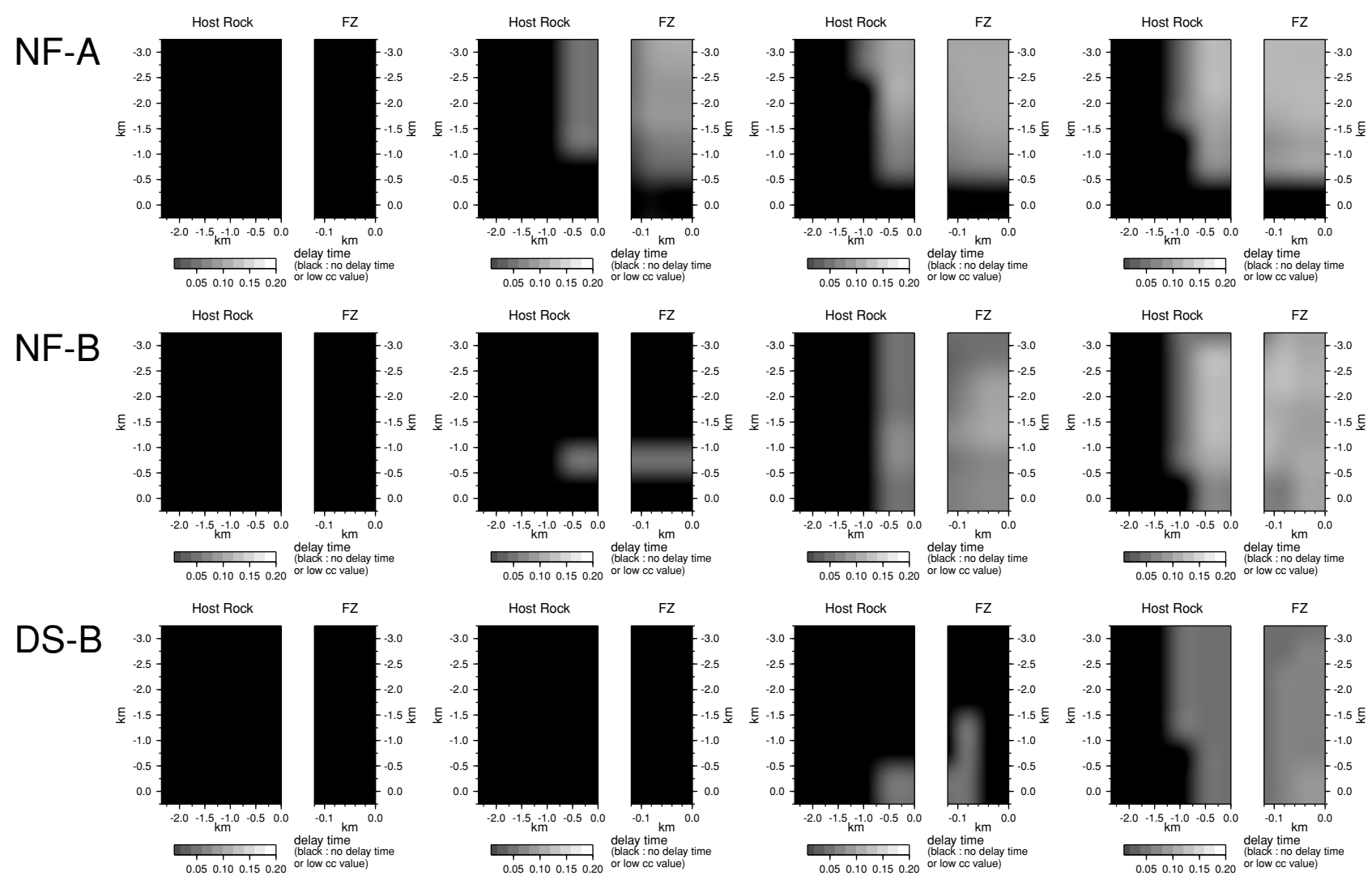

Fig. D3. Measured delay time between arrivals of $q S_{1}-$ and $q S_{2}$ wave for NF-A, -B, and DS-B sources located outside the fault zone. Other notations are the same as in Fig. 5.

$$
\boldsymbol{b}\left(z_{0}\right)=\frac{-1}{R_{55}}\left[\begin{array}{c}
X_{5 i} S_{i} \\
0
\end{array}\right]
$$

(Mandal and Mitchell, 1986), while imposing the radiation boundary condition at $z=z_{0}$ and $z_{N-1}$, we can derive the displacement-stress vector at $z_{0}$ as:

$$
\begin{gathered}
\boldsymbol{b}\left(z_{0}\right)=\frac{-1}{W_{11} W_{22}-W_{12} W_{21}} \times \\
{\left[\begin{array}{l}
E_{11}\left(W_{22} X_{1 i} S_{i}-W_{12} X_{2 i} S_{i}\right)+E_{12}\left(-W_{21} X_{1 i} S_{i}+W_{11} X_{2 i} S_{i}\right) \\
E_{21}\left(W_{22} X_{1 i} S_{i}-W_{12} X_{2 i} S_{i}\right)+E_{22}\left(-W_{21} X_{1 i} S_{i}+W_{11} X_{2 i} S_{i}\right) \\
E_{31}\left(W_{22} X_{1 i} S_{i}-W_{12} X_{2 i} S_{i}\right)+E_{32}\left(-W_{21} X_{1 i} S_{i}+W_{11} X_{2 i} S_{i}\right) \\
E_{41}\left(W_{22} X_{1 i} S_{i}-W_{12} X_{2 i} S_{i}\right)+E_{42}\left(-W_{21} X_{1 i} S_{i}+W_{11} X_{2 i} S_{i}\right)
\end{array}\right]}
\end{gathered}
$$$$
i=1,2,3,4, \quad(P-S V)
$$

$$
\boldsymbol{b}\left(z_{0}\right)=\frac{-1}{W_{55}}\left[\begin{array}{l}
E_{55}\left(S_{5} X_{55}+S_{6} X_{56}\right) \\
E_{65}\left(S_{5} X_{55}+S_{6} X_{56}\right)
\end{array}\right](S H)
$$

(Nakamura and Takenaka, 2005), where

$$
\boldsymbol{W}=\boldsymbol{D}_{N}^{-1} \boldsymbol{P}_{N-1} \cdots \boldsymbol{P}_{1} \boldsymbol{D}_{1} .
$$

By using the above equations and transforming the coordinates as shown in Fig. A2, synthetic seismograms of waves propagating through a vertically layered medium can be calculated.

\section{Appendix B. Source Vector for Dislocation Source of Unit Potency}

Mandal and Mitchell (1986) described the formulation for double couples with unit moment. Here we show the corresponding formulation for dislocation source of unit potency. For dislocation source of unit potency, the source matrices $S^{m}$ in Appendix B in Mandal and Mitchell (1986) should be replaced by

$$
\begin{aligned}
& S^{0}=\left[0,-F / C+1,0,-k\left(A-\left(F^{2} / C\right)-N\right), 0,0\right]^{T}, \\
& S^{1}=[1,0,0,0,-1,0]^{T}, \\
& S^{2}=[0,0,0,-k N, 0, k N]^{T},
\end{aligned}
$$

and the vector $\left(v_{1}, v_{2}, v_{3}\right)$ in their Eqs. (20a) to (20c) should also be replaced by the unit vector in the direction of the slip instead of the unit vector in the direction of the force. Equation (B.1) can be derived by rearranging equation (245) in Takeuchi and Saito (1972) along with the formulation of Mandal and Mitchell (1986).

\section{Appendix C. Synthetic Seismograms of the Source Located Outside the Fault Zone}

We show synthetic seismograms of SS-A, -B, NF-A, -B, and DS-A sources in Fig. C1. 
Appendix D. Results of the Source Located Outside the Fault Zone with the CrossCorrelation Method

We show the results of measuring shear-wave splitting parameters with the cross-correlation method for all sources located outside the fault zone in Figs. D1, D2 and D3.

\section{References}

Anderson, D. L. and A. M. Dziewonski, Upper mantle anisotropy: evidence from free oscillations, Geophys. J. Roy. Astr. Soc., 69, 383-404, 1982.

Ben-Menahem, A, Source mechanism of the 1906 San Francisco earthquake, Phys. Earth Planet. Inter., 17, 163-181, 1978.

Booth, D. C. and S. Crampin, Shear-wave polarizations on a curved wavefront at an isotropic free-surface, Geophys. J. Roy. Astr, Soc., 83, 31-45, 1985.

Bowman, J. R. and M. Ando, Shear-wave splitting in the uppermantle wedge above the Tonga subduction zone, Geophys. J. Roy. Astr. Soc., 88, 25-41, 1987 .

Chester, F. M., J. P. Evans, and R. L. Biegel, Internal structure and weakening mechanisms of the San Andreas fault, J. Geophys. Res., 98, 771$786,1993$.

Cochran, E. S., J. E. Vidale, and Y. G. Li, Near-fault anisotropy following the Hector Mine earthquake, J. Geophys. Res., 108, 2436, doi:10.1029/ 2002JB002352, 2003

Crampin, S., Seismic wave propagation through a cracked solid : polarization as a possible dilatancy diagnostic, Geophys. J. Roy. Astr. Soc., 53, 467-496, 1978

Crampin, S. and S. Peacock, A review of shear-wave splitting in the compliant crack-critical anisotropic Earth, Wave Motion, 41, 59-77, 2005.

Crampin, S., R. Evans, B. Üçer, M. Doyle, J. P. Davis, G. V. Yegorkina, and A. Miller, Observations of dilatancy-induced polarization anomalies and earthquake prediction, Nature, 286, 874-877, 1980.

Evans, R., Effects of the free surface on shear wavetrains, Geophys. J. Roy. Astr. Soc., 76, 165-172, 1984.

Gilbert, F. and G. Backus, Propagator matrices in elastic wave and vibration problems, Geophysics, 31, 326-332, 1966.

Haskell, N. A., The dispersion of surface waves in multilayered media, Bull. Seism. Soc. Am., 43, 17-34, 1953.

Herrmann, R. B., SH-wave generation by dislocation sources-A numerical study, Bull. Seism. Soc. Am., 69, 1-15, 1979.

Hough, S. E., Y. Ben-Zion, and P. C. Leary, Fault-zone waves observed at the southern Joshua Tree earthquake rupture zone, Bull. Seism. Soc. Am., 84, 761-767, 1994.

Hudson, J. A., Overall properties of a cracked solid, Math. Proc. Camb. Phil. Soc., 88, 371-384, 1980.

Hudson, J. A., Wave speeds and attenuation of elastic waves in material containing cracks, Geophys. J. Roy. Astr. Soc., 64, 133-150, 1981.

Igel, H., Y. Ben-zion, and P. C. Leary, Simulation of SH- and P-SV-wave propagation in fault zones, Geophys. J. Int., 128, 533-546, 1997.

Kawasaki, I. and T. Tanimoto, Radiation patterns of body waves due to the seismic dislocation occurring in an anisotropic source medium, Bull. Seism. Soc. Am., 71, 37-50, 1981.
Li, Y. G., P. C. Leary, K. Aki, and P. E. Malin, Seismic trapped modes in the Oroville and San Andreas fault zones, Science, 249, 763-766, 1990.

Li, Y. G., W. L. Ellsworth, C. H. Thurber, P. E. Malin, and K. Aki, Faultzone guided waves from explosions in the San Andreas fault at Parkfield and Cienega Valley, California, Bull. Seism. Soc. Am., 87, 210-221, 1997.

Liu, E. and S. Crampin, Effects of the internal shear wave window: Comparison with anisotropy induced splitting, J. Geophys. Res., 95, 1127511281, 1990.

Mandal, B. and B. J. Mitchell, Complete seismogram synthesis for transversely isotropic media, J. Geophys., 59, 149-156, 1986.

Mizuno, T., K. Yomogida, H. Ito, and Y. Kuwahara, Spatial distribution of shear wave anisotropy in the crust of the southern Hyogo region by borehole observation, Geophys. J. Int., 147, 528-542, 2001.

Nakamura, T. and H. Takenaka, Influence of anisotropy in the fault zone on the seismic wave, Zisin 2 (J. Seism. Soc. Jpn.), 57, 331-342, 2005 (in Japanese with English abstract).

Nakamura, T., H. Takenaka, and S. Suzuki, Strong $S$-wave anisotropy in the aftershock region of the 2000 Tottori-ken Seibu, Japan, earthquake $\left(M_{\mathrm{w}} 6.6\right)$, Earth Planets Space, 57, 1055-1062, 2005.

O'Connell, R. and B. Budiansky, Seismic velocities in dry and saturated cracked solids, J. Geophys. Res., 79, 5412-5426, 1974.

Peacock, S., S. Crampin, D. C. Booth, and J. B. Fletcher, Shear-wave splitting in the Anza seismic gap, Southern California: temporal variations as possible precursors, J. Geophys. Res., 93, 3339-3356, 1988.

Peng, Z. and Y. Ben-Zion, Systematic analysis of crustal anisotropy along the Karadere-Düzce branch of the north Anatolian fault, Geophys. $J$. Int., 159, 253-274, 2004.

Savage, M. K., Seismic anisotropy and mantle deformation: What have we learned from shear wave splitting?, Rev. Geophys, 37, 65-106, 1999.

Shih, X. R., R. P. Meyer, and J. F. Schneider, An automated, analytical method to determine shear-wave splitting, Tectonophysics, 165, 271$278,1989$.

Takeuchi, H. and M. Saito, Seismic surface waves, in Methods in Computational Physics, Vol. 11, pp. 217-295, edited by B. A. Bolt, Academic Press, New York, 1972.

Vavryčuk, V., Inversion for anisotropy from non-double-couple components of moment tensors, J. Geophys. Res., 109, B07306, doi:10.1029/ 2003JB002926, 2004

Wang, C. Y. and R. B. Herrmann. A numerical study of P-, SV-, and SHwave generation in a plane layered medium, Bull. Seism. Soc. Am., 70, 1015-1036, 1980.

Watanabe, A., H. Takenaka, and S. Suzuki, Spatial variation of shear wave anisotropy in the focal region of the 1997 northwestern Kagoshima earthquakes, Abstr. Jpn. Earth Planet. Sci. Joint Meeting, Sz-P003, 2001

Wessel, P. and W. H. F. Smith, Free software helps map and display data, EOS Trans. Am. Geophys. Union, 72, 441-446, 1991.

Yamanaka, H., Y. Hiramatsu, and H. Katao, Spatial distribution of atypical aftershocks of the 1995 Hyogo-ken Nanbu earthquake, Earth Planets Space, 54, 933-945, 2002.

T. Nakamura (e-mail: nakamura@geo.kyushu-u.ac.jp) and H. Takenaka (e-mail: takenaka@geo.kyushu-u.ac.jp) 